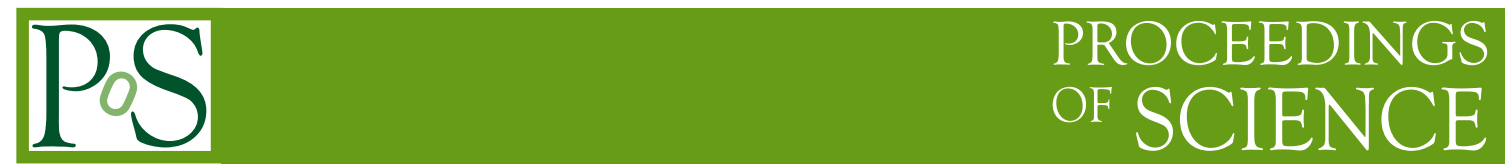

\title{
Latest Results of the MEG Experiment
}

\author{
Fabrizio Cei ${ }^{* \dagger}$ \\ INFN and Università di Pisa, Dipartimento di Fisica, Largo Bruno Pontecorvo 3, 56127 Pisa, \\ Italy \\ E-mail: fabrizio.cei@pi.infn.it
}

We present recent results of the MEG experiment, which has been searching for the Lepton Flavour Violating decay $\mu^{+} \rightarrow \mathrm{e}^{+} \gamma$ since several years. In a combined dataset corresponding to $3.6 \times 10^{14}$ stopped muons on target we didn't find any evidence for this decay and we established an upper bound on the $\mu^{+} \rightarrow \mathrm{e}^{+} \gamma$ branching ratio of $5.7 \times 10^{-13}$ at $90 \%$ C.L., which improves our world best limit by a factor four. We discuss also the expected final sensitivity of the MEG experiment.

The European Physical Society Conference on High Energy Physics -EPS-HEP2013

18-24 July 2013

Stockholm, Sweden

\footnotetext{
* Speaker.

$\dagger$ On behalf of the MEG Collaboration.
} 


\section{Introduction to Lepton Flavour Violation and $\mu^{+} \rightarrow \mathrm{e}^{+} \gamma$ decay}

The Lepton Flavour Violation (LFV) in the charged lepton sector is almost forbidden in the Standard Model (SM), even including neutrino oscillations and mixing [1]. Conversely, the majority of new physics models [2] Beyond SM (BSM), particularly in view of recent measurements of a large $\theta_{13}$ at reactor [3] and accelerator [4] experiments, predict measurable branching ratios $(\mathscr{B})$ for LFV reactions, as $\mu^{+} \rightarrow \mathrm{e}^{+} \gamma$. The discovery of a LFV process would be a clear evidence for BSM physics, whereas improvements in the $\mathscr{B}$ upper limits (UL) constitute significant constraints on the parameter space, complementary to those obtainable at high energy colliders. The present best UL on the $\mu^{+} \rightarrow \mathrm{e}^{+} \gamma$ decay, $\mathscr{B}<2.4 \times 10^{-12}$ at $90 \%$ C.L., was set by the MEG experiment [5] with an analysis of the data taken in the years $2009-2010$, for a total number of $1.75 \times 10^{14}$ $\mu^{+}$stopped on target. Here we present an updated analysis of the $2009-2010$ data sample, based on recently improved algorithms for the reconstruction of positrons and photons together with the analysis of the data sample collected in 2011 , which corresponds to $1.85 \times 10^{14} \mu^{+}$stopped on target. The combined analysis of the full $2009-2011$ statistics is also reported.

\section{The MEG Experiment}

The MEG experimet at PSI (Paul Scherrer Institute [6]) aims to search for the $\mu^{+} \rightarrow \mathrm{e}^{+} \gamma$ decay with a sensitivity $\sim 10^{-13}$. The $\mu^{+} \rightarrow \mathrm{e}^{+} \gamma$ signature for muons decaying at rest is a back-toback monoenergetic (52.83 MeV each) time-coincident $\mathrm{e}^{+}-\gamma$ pair. Positron and photon candidates are characterized by their energies $\left(E_{\gamma}, E_{\mathrm{e}}\right)$, their relative directions $\left(\theta_{\mathrm{e} \gamma}, \phi_{\mathrm{e} \gamma}\right)^{1}$ and emission time $\left(t_{\mathrm{e} \gamma}\right)$. The background has two components: 1) the Radiative Muon Decay $\mu^{+} \rightarrow \mathrm{e}^{+} v_{\mathrm{e}} \bar{v}_{\mu} \gamma$ (RMD), whose rate is proportional to the muon stopping frequency $\left.R_{\mu} ; 2\right)$ the ACCidental Background (ACCB), given by the random coincidence of energetic positrons from the SM Michel decay with photons from RMD, $\mathrm{e}^{+}-\mathrm{e}^{-}$annihilation-in-flight or bremsstrahlung. The ACCB, whose rate is proportional to $R_{\mu}^{2}$, is responsible of $93 \%$ of MEG events with $E_{\gamma}>48 \mathrm{MeV}$. The MEG experiment [7] uses the PSI $\pi$ E5 beam line, capable of delivering up to $10^{8}$ stopping $\mu^{+} / \mathrm{s}$, even if a lower intensity of $3 \times 10^{7} \mu^{+} / \mathrm{s}$ is used to take ACCB under control. The experiment employs a Liquid Xenon (LXe) detector for the photon and a magnetic spectrometer for the positron measurement. The photon detector is formed by a single volume $(\sim 900 \ell)$ of LXe, viewed by 846 UV-sensitive photomultiplier tubes (PMTs) submerged in the liquid. The spectrometer is composed by $16 \mathrm{Drift}$ Chambers (DC), each one formed by two staggered layers of sense wires and cathodic foils, and by a double-array of scintillation Timing Counter (TC), located inside a superconducting solenoid (COnstant Bending RAdius, COBRA) with a gradient magnetic field along the beam axis. The magnetic field is arranged to sweep out positrons with small longitudinal momenta and make the positron bending radius almost independent of emission angle. The detector is continuously monitored by a multi-element calibration system [5, 7, 8] including: 1 ) a Cockroft-Walton accelerator; 2) an array of $\alpha$ sources mounted inside the photon detector; 3) a liquid hydrogen target, used in conjunction with a $\pi^{-}$beam to induce the Charge Exchange (CEX) reaction $\pi^{-} \mathrm{p} \rightarrow \pi^{0} \mathrm{n}$, followed by the $\pi^{0}$ decay in two photons; 4) a variable monoenergetic positron beam and 5) a neutron

\footnotetext{
${ }^{1} \theta_{\mathrm{e} \gamma}=\left(\pi-\theta_{\mathrm{e}}\right)-\theta_{\gamma}$ and $\phi_{\mathrm{e} \gamma}=\left(\pi+\phi_{\mathrm{e}}\right)-\phi_{\gamma}, \theta$ and $\phi$ being the polar angle and the azimuthal angle respectively, taking the beam-axis as $z$-axis.
} 
generator. In the CEX calibration the NaI detector, used to define the back-to-back two-photon coincidence with the LXe detector, was recently replaced by a higher resolution BGO array detector. The relative alignment between the DCs and between the LXe detector and the spectrometer is obtained by looking at straight tracks from cosmic muons and at curved Michel positron tracks and comparing the measurements with an optical survey, improved in 2011 by inserting a laser tracker and prismatic corner cube reflectors mounted on the DC modules. Finally, in 2011 the DAQ efficiency, defined as the product of DAQ live time fraction and trigger efficiency to select signal event, increased from $\approx 72 \%$ to $\approx 96 \%$ thanks to a new multiple buffer read out scheme [9].

\section{Analysis procedure, improvements and results}

Our analysis is based on a maximum likelihood (ML) technique applied in the analysis region defined by $48 \mathrm{MeV}<E_{\gamma}<58 \mathrm{MeV}, 50 \mathrm{MeV}<E_{\mathrm{e}}<56 \mathrm{MeV},\left|t_{\mathrm{e} \gamma}\right|<0.7 \mathrm{~ns},\left|\theta_{\mathrm{e} \gamma}\right|<50 \mathrm{mrad}$ and $\left|\phi_{\mathrm{e} \gamma}\right|<50 \mathrm{mrad}$, described in [5]. We call "time sidebands" the regions defined by $1 \mathrm{~ns}<\left|t_{\mathrm{e} \gamma}\right|<$ $4 \mathrm{~ns}$, " $E_{\gamma}$-sideband" that defined by $40 \mathrm{MeV}<E_{\gamma}<48 \mathrm{MeV}$ and "angle sidebands" those defined by $50 \mathrm{mrad}<\left|\phi_{\mathrm{e} \gamma}\right|<150 \mathrm{mrad}$ or $50 \mathrm{mrad}<\left|\theta_{\mathrm{e} \gamma}\right|<150 \mathrm{mrad}$. The positron track is reconstructed by combining its measured positions at each DC layer (hit), while longitudinal $z$-positions are derived from signals induced on the segmented DC cathodes. A new reconstruction algorithm, based on a fast Fourier transform filtering technique, was developed and applied to mitigate the $z$-resolution degradation due to the electromagnetic noise, yielding an up to $\sim 10 \%$ improvement in angular resolution. The positron kinematic variables are extracted by using a Kalman filter track fitting technique [10], completely revised for this analysis to include a better model for the hits and the track itself, based on the GEANE package [11]. An improved model for multiple scattering and energy loss in the detector materials and a detailed map of the magnetic field, measured with a $0.2 \%$ precision, were also included. The fitted positron track is propagated to the TC allowing an iterative refinement of the hits with the positron time measurement. The track fit yields a parameter covariance matrix, resulting in very good agreement with the measured resolutions, extracted by using the two turn method [5]. Consequently a per-track error is determined which allows us to follow the variable DC performance during the data-taking period and is taken into account in the ML analysis. The average hit multiplicity for a track is $\approx 10$ and only tracks with $\geq 7$ hits and $\leq 2$ turns in the spectrometer are retained for the analysis; a single positron per event is selected by applying additional track quality cuts. The improvement in positron reconstruction with respect to the previous analysis can be seen in Fig. 1 (left) where the reconstructed positron energy near the kinematic edge of the Michel decay spectrum shows a reduced tail. The energy resolution, evaluated by fitting the kinematic edge, is well described by the sum of three Gaussian curves with a resolution $\sigma_{E_{\mathrm{e}}}=305 \mathrm{keV}$ for the core component (85\%). The $\phi_{\mathrm{e}}$-resolution has a $\phi_{\mathrm{e}}$-dependence with a minimum at $\phi_{\mathrm{e}}=0$, where it is measured by the two-turn method to be $\sigma_{\phi_{\mathrm{e}}}=7.5(7.0) \mathrm{mrad}^{2}$. Similarly the measured $\theta_{\mathrm{e}}$-resolution is $\sigma_{\theta_{\mathrm{e}}}=10.6(10.0) \mathrm{mrad}$. The decay vertex coordinates and the positron direction at the vertex are determined by extrapolating the reconstructed track back to the target. The resolutions on the decay vertex coordinates, also determined by the two-turn method, are described by a Gaussian curve with $\sigma_{z}=1.9(1.5) \mathrm{mm}$ and, in the vertical direction,

\footnotetext{
${ }^{2}$ From here on we will quote in parentheses the value in the 2009-2010 data when different from that in 2011.
} 

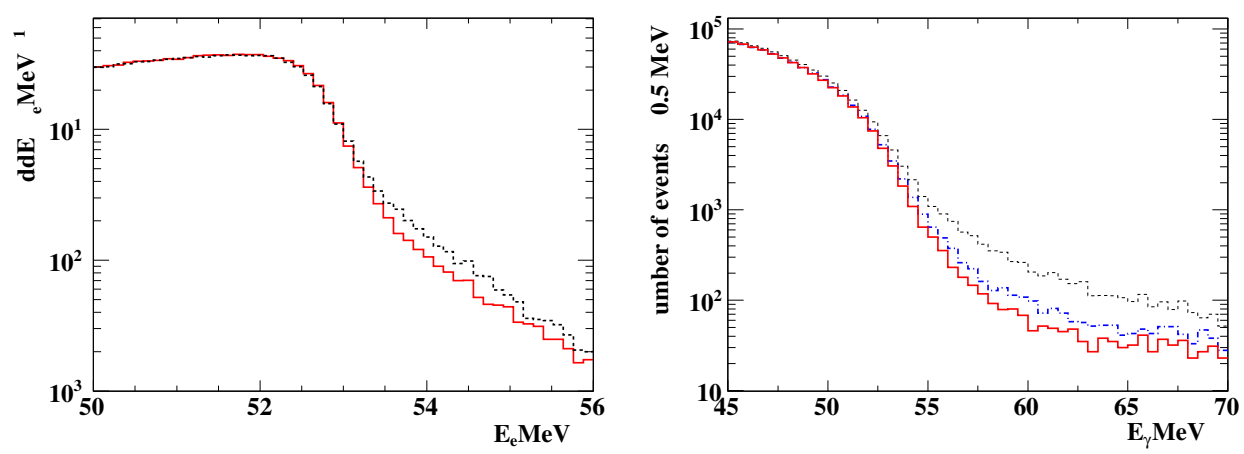

Figure 1: (Left) The Michel positron energy spectrum in the 2010 dataset time sidebands with the old (black dashed line) and the new (red solid line) track reconstruction code. (Right) The photon background energy spectra in the 2010 dataset time sidebands with different pile-up elimination algorithms. Black dots: no pile-up elimination; blue dot-dashed line: previous algorithm; red solid line: new algorithm.

by the sum of two Gaussian curves with $\sigma_{y}=1.3(1.2) \mathrm{mm}$ for the core component (85\%). The LXe detector uses the xenon scintillation light to measure the total energy released by the photon, the position and the time of its first interaction. The 3-D photon interaction point is reconstructed by using the pattern of scintillation light detected by the PMTs near the incident position and the photon interaction time by combining the leading edge times of the PMT waveforms. The photon direction is defined by the line connecting the decay vertex to the photon interaction point in the LXe detector. The photon energy reconstruction is based on the sum of the charges collected by all PMTs. Monochromatic $55 \mathrm{MeV}$ photons from $\pi^{0}$ decay are used to determine the absolute energy scale. Pile-up photons, which at $R_{\mu}=3 \times 10^{7} \mu^{+} / \mathrm{s}$ affect $\approx 15 \%$ of triggered events, were identified, in the previous analyses, topologically by the PMT light distribution and temporally by the PMT leading edge time distribution. In addition to these methods a new algorithm, analyzing waveforms after summing up all channels at the end of the full chain of photon reconstruction, was developed. This algorithm allows also to re-adjust the charge integration window for the energy estimate, resulting in a better energy reconstruction. The improvement can be seen in Fig. 1 (right) where the photon energy spectrum shows a smaller high-energy tail when the new pile-up rejection algorithm is applied. The efficiency of photon reconstruction increased from 59\% to $63 \%$ due to the new algorithm. The position reconstruction resolution, evaluated by a MC simulation and validated in CEX runs by placing lead slit collimators in front of the LXe detector, is $5 \mathrm{~mm}$ on the photon entrance face and $6 \mathrm{~mm}$ along the radial depth $w$. The timing and energy resolutions are evaluated using 55 and $83 \mathrm{MeV}$ photons from $\pi^{0}$ decay. The LXe timing resolution is $\sigma_{t_{\gamma}}=67 \mathrm{ps}$ at $52.8 \mathrm{MeV}$. The position-dependent energy resolutions are measured in the CEX data and the average energy resolution, extracted from a Gaussian fit to the high energy side of the spectrum, results $1.7 \%(1.9 \%)$ and $2.4 \%(2.4 \%)$ for $w>2 \mathrm{~cm}$ and $w<2 \mathrm{~cm}$ respectively. The resolutions of the relative directions are obtained by combining the relevant resolutions of positrons and photons discussed above. The results are 16.2 (15.7) $\mathrm{mrad}$ for $\theta_{\mathrm{e} \gamma}$ and 8.9 (9.0) $\mathrm{mrad}$ for $\phi_{\mathrm{e} \gamma}$. The relative time $t_{\mathrm{e} \gamma}$ is derived from the time measurements in the LXe detector and in the TC, after correcting 
for the particle time-of-flights. The corresponding resolution at $52.8 \mathrm{MeV}$ energy is 127 (135) ps, measured from the RMD peak observed in the $E_{\gamma}$-sideband above the flat ACCB distribution; a small correction takes into account the $E_{\gamma}$-dependence measured in the CEX runs. The position of the RMD-peak ( $\left.t_{\mathrm{e} \gamma}=0\right)$, monitored constantly during the data-taking period, is stable within $15 \mathrm{ps}$.

The ML analysis has been supplemented by a blind analysis procedure for the 2011 dataset, which involved masking a region of $48 \mathrm{MeV}<E_{\gamma}<58 \mathrm{MeV}$ and $\left|t_{\mathrm{e} \gamma}\right|<1$ ns until the Probability Density Functions (PDFs) for the likelihood function are finalized. The time and angle sidebands are used to optimize the analysis, to study ACCB and to extract the corresponding PDFs. Calibration data and measured resolutions are used to build the Signal (S) and RMD events PDFs. Different resolutions and correlations are included in the PDFs on an event-by-event basis. The dependence on the photon interaction point and the quality of the positron tracking has already been incorporated into the PDFs, while in the new analysis the per-event positron error matrix, estimated by the new Kalman filter, has been introduced. All the PDFs are inserted in the global likelihood function (defined in [5]) which includes also the sideband constraints on the number of RMD and ACCB events. The ML fit is performed to estimate the number of S, RMD and ACCB events in the analysis region. An analysis with constant PDFs is also performed as a crosscheck, showing consistent results. The confidence interval for the number of $\mathrm{S}$ events is calculated by a frequentist method with a profile likelihood-ratio ordering [5, 12], where the numbers of RMD and ACCB events are treated as nuisance parameters. Applying the same procedure to a large sample of simulated experiments (toy MCs), generated according to the experimental PDFs and background rates and with $S=0$ hypothesis, we evaluated our sensitivity $\mathscr{S}_{90}$, defined as the median of the $90 \%$ C.L. ULs extracted from the toy MC ensemble. Likelihood fits are also performed in fictitious analysis regions in the time- and angle-sidebands, getting ULs in good agreement with the $\mathscr{S}_{90}$ 's. The normalization factor needed to convert an UL on the number of S events into an UL on $\mathscr{B}\left(\mu^{+} \rightarrow \mathrm{e}^{+} \gamma\right)$ is computed by using two independent schemes, either counting the number of Michel positrons selected with a dedicated pre-scaled trigger, or the number of RMD events observed in the muon data. The combined uncertainty on the $\mathscr{B}$ value is $4 \%$. The increased reconstruction efficiency of the new algorithms results in a $14 \%$ larger data sample for the $\mu^{+} \rightarrow \mathrm{e}^{+} \gamma$ search. The systematic uncertainties on the PDF parameters and on the normalization are taken into account in the calculation of the confidence intervals by fluctuating the PDFs by the amount of the uncertainties. The global effect on the observed UL is $1 \%$, with the main contribution coming from the angular PDFs.

Fig. 2 shows the event distributions in the $\left(E_{\mathrm{e}}, E_{\gamma}\right)$ - and $\left(\cos \Theta_{\mathrm{e} \gamma}, t_{\mathrm{e} \gamma}\right)$-planes for the combined $2009-2011$ dataset, where $\Theta_{\mathrm{e} \gamma}$ is the opening angle between positron and photon, together with the contours of the averaged signal PDFs. We show in Fig. 3 (left) the results of the ML fit for the full dataset 2009-2011: the best fit value for the number of $S$ events is -0.4 . The observed profile likelihood ratios as a function of $\mathscr{B}$ are shown in Fig. 3 (right). The best $\mathscr{B}$ fit $\left(\mathscr{B}_{\mathrm{fit}}\right), \mathrm{UL}$ at $90 \%$ C.L. $\left(\mathscr{B}_{90}\right)$ and $\mathscr{S}_{90}$ for the combined $2009-2010$ dataset, the 2011 data alone and the full $2009-$ 2011 dataset are listed in Table 1 . The $\mathscr{B}_{90}$ for the latter is $5.7 \times 10^{-13}$. As a quality check the ML fit was repeated for the $2009-2011$ dataset omitting the constraint on the number of background events. We obtained $N_{\mathrm{RMD}}=163 \pm 32$ and $N_{\mathrm{ACCB}}=2411 \pm 57$, in good agreement with the expectations estimated from $E_{\gamma}$ and time sidebands, $\left\langle N_{\mathrm{RMD}}\right\rangle=169 \pm 17$ and $\left\langle N_{\mathrm{ACCB}}\right\rangle=2415 \pm 25$. The reanalysis of the $2009-2010$ dataset with new algorithms caused variations in the values of the observables much smaller than the detector resolutions, which, however, induced a change 

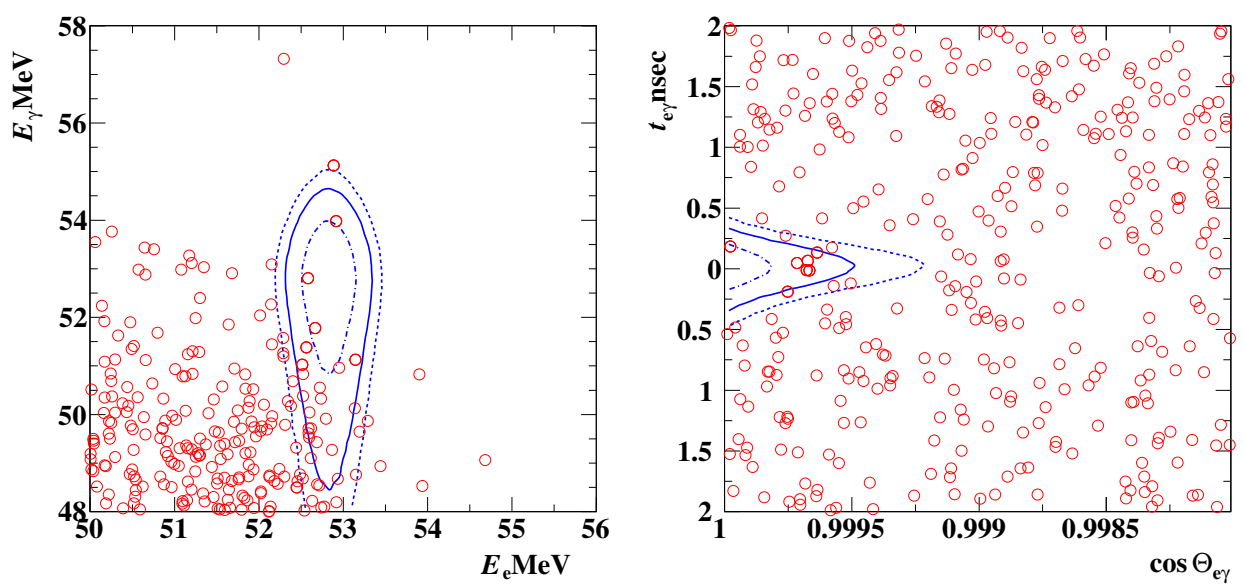

Figure 2: Event distributions for the combined $2009-2011$ dataset in the $\left(E_{\mathrm{e}}, E_{\gamma}\right)$ - and $\left(\cos \Theta_{\mathrm{e} \gamma}, t_{\mathrm{e} \gamma}\right)$-planes. In the left (right) panel, a selection of $\left|t_{\mathrm{e} \gamma}\right|<0.244 \mathrm{~ns}$ and $\cos \Theta_{\mathrm{e} \gamma}<-0.9996$ with $90 \%$ efficiency for each variable $\left(52.4 \mathrm{MeV}<E_{\mathrm{e}}<55 \mathrm{MeV}\right.$ and $51 \mathrm{MeV}<E_{\gamma}<55.5 \mathrm{MeV}$ with $90 \%$ and $74 \%$ efficiencies for $E_{\mathrm{e}}$ and $E_{\gamma}$, respectively) is applied. The signal PDF contours (1, 1.64 and $2 \sigma$ ) are also shown.
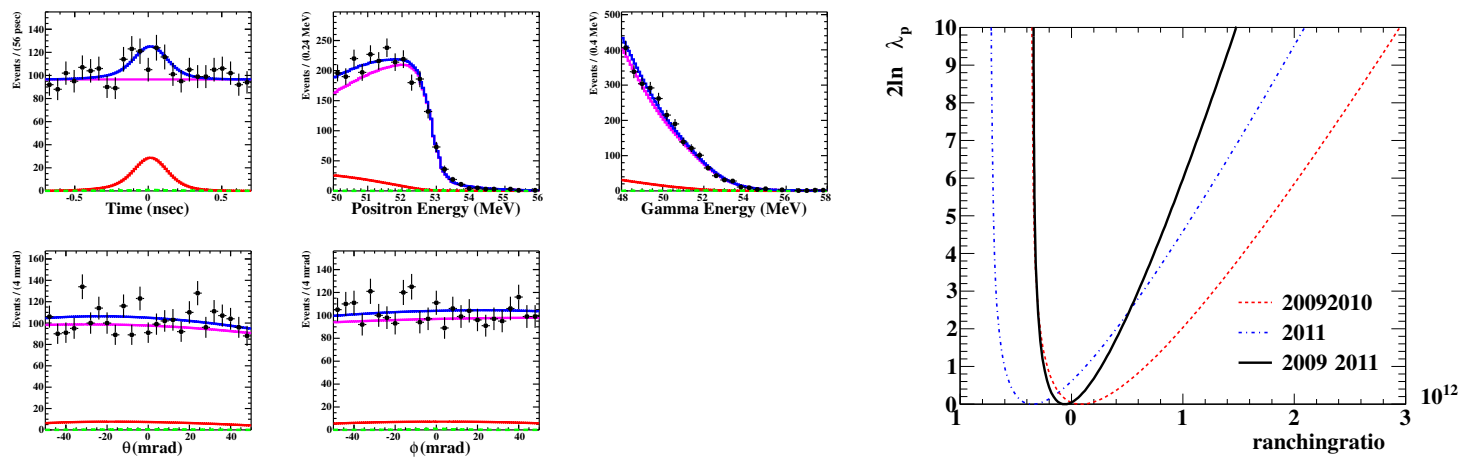

Figure 3: Left: results of ML fit for the full 2009 - 2011 dataset. The black dots are the data and the colour lines represent the contributions extracted from the fit: $\mathrm{S}$ (green), RMD (red), ACCB (purple) and total (blue). Right: observed profile likelihood ratios $\left(\lambda_{\mathrm{p}}\right)$ as a function of the $\mathscr{B}$ for the $2009-2010$ combined data, the 2011 data alone and the combined 2009 - 2011 data sample.

in $\mathscr{S}_{90}, \mathscr{B}_{\text {fit }}$ and $\mathscr{B}_{90}$ for the same dataset. The sensitivity for this dataset in previous analysis was $1.6 \times 10^{-12}$ and the observed $\approx 20 \%$ improvement is in agreement with the expectations. We compared $\mathscr{B}_{90}$ 's obtained with the new and old analyses for the same sample of simulated experiments and found that the probability of a change of $\mathscr{B}_{90}$ at least equal to that observed in the $2009-2010$ dataset is $31 \%$. The UL obtained from the 2011 data only is more stringent than $\mathscr{S}_{90}$. However, this is not unusual, since the probability of having $\mathscr{B}_{90}$ equal or smaller than that observed in the 2011 data is calculated to be $24 \%$ with a sample of toy MC experiments.

\section{Summary and future perspectives}

On the basis of data collected between 2009 and 2011 the MEG experiment established the 


\begin{tabular}{lccc} 
Dataset & $\mathscr{B}_{\text {fit }} \times 10^{12}$ & $\mathscr{B}_{90} \times 10^{12}$ & $\mathscr{S}_{90} \times 10^{12}$ \\
\hline \hline $2009-2010$ & 0.09 & 1.3 & 1.3 \\
2011 & -0.35 & 0.67 & 1.1 \\
$2009-2011$ & -0.06 & 0.57 & 0.77 \\
\hline \hline
\end{tabular}

Table 1: Best fit values $\left(\mathscr{B}_{\text {fit }}\right.$ 's), branching ratio upper limit $\left(\mathscr{B}_{90}\right)$ and sensitivities $\left(\mathscr{S}_{90}\right)$

most stringent UL to date on the $\mu^{+} \rightarrow \mathrm{e}^{+} \gamma$ decay: $\mathscr{B}<5.7 \times 10^{-13}$ at $90 \%$ C.L., which improves our previous UL by a factor four [13] and is 20 times better than the best upper bound of pre-MEG era [14]. Other data have also been acquired in 2012 and 2013 and the total number of stopped $\mu^{+}$is expected to be almost twice that of the sample analyzed so far; then we estimated a final sensitivity of our experiment of $\sim 5 \times 10^{-13}$. Our result represents an important constraint for BSM models, which will be improved at the end of data analysis. The collaboration is also working on the project of an upgraded detector [15], discussed in another talk at this conference [16].

\section{References}

[1] F. Böhm \& P. Vogel, Physics of Massive Neutrinos, Cambridge University Press, (1992), S. T. Petcov, Sov. J. Nucl. Phys. 25 (1977) 340.

[2] R. Barbieri, L. Hall and A. Strumia, Nucl. Phys. B 455 (1995) 219, J. Hisano, D. Nomura and T. Yanagida, Phys. Lett. B 437 (1998) 351, M. Raidal et al., Eur. Phys. J. C 57 (2008) 13, G. Blankenburg et al., Eur. Phys. J. C 72 (2012) 2126.

[3] F. P. An et al., Phys. Rev. Lett. 108 (2012) 171803, J. K. Ahn et al., Phys. Rev. Lett. 108 (2012) 191802, Y. Abe et al., Phys. Lett. B 723 (2013) 66.

[4] K. Abe et al., Phys. Rev. Lett. 107 (2011) 041801.

[5] J. Adam et al., Phys. Rev. Lett. 107 (2011) 171801.

[6] http://www.psi.ch

[7] J. Adam et al., Eur. Phys. J. C 73 (2013) 2365

[8] J. Adam et al., Nucl. Instr. Meth. A 641 (2011) 19, A. M. Baldini et al., Nucl. Instr. Meth. A 565 (2006) 589, A. M. Baldini et al., Nucl. Instr. Meth. A 545 (2006) 753

[9] L. Galli et al., JINST 8 (2013) P01008.

[10] P. Billoir, Nucl. Instr. Meth. A 225 (1984) 352, R. Frühwirth, Nucl. Instr. Meth. A 262 (1987) 444.

[11] V. Innocente and E. Nagy, Nucl. Instr. Meth. A 324 (1993) 297, A. Fontana et al., J. Phys. Conf. Ser. 119 (2008) 032018.

[12] J. Beringer et al. (Particle Data Group), Phys. Rev. D 86 (2012) 010001, G. J. Feldman and R.D. Cousins, Phys. Rev. D 57 (1998) 3873.

[13] J. Adam et al., Phys. Rev. Lett. 110 (2013) 201801

[14] M. Ahmed et al., Phys. Rev. D. 65 (2002) 112002.

[15] A. M. Baldini et al., MEG Upgrade Proposal, physics .ins-det/1301.7225

[16] Y. Uchiyama, these proceedings. 